\title{
FEMORAL FRACTURES PRODUCED BY PROJECTILES - THE EFFECTS OF MASS AND DIAMETER ON TARGET DAMAGE*†
}

\author{
JAMES H. HARGER and DONALD F. HUELKE \\ Department of Anatomy, The University of Michigan Medical School, Ann Arbor, \\ Mich. 48104, U.S.A.
}

\begin{abstract}
In order to determine the relative importance of projectile mass and diameter in the production of damage to human bone, a total of 560 embalmed human femurs were impacted by spheres of varying diameter and specific gravity. The results strongly support the conclusion that, at a given impact velocity, projectile diameter is a far greater factor influencing energy expenditure and thus damage to the target than the mass of the projectile. Thus a large, lightweight projectile will be more destructive to bone, and probably to all tissues, than a smaller but more massive one.
\end{abstract}

\section{INTRODUCTION}

THE EFFECTIVENESS of a projectile in producing damage depends on the amount of energy imparted to the target at impact, for the more energy transferred from the projectile to the target the greater the damage caused. The effect of projectile impacts on human bone has not been extensively studied prior to our series (Huelke et al., 1968a and 1968b), although there are several published investigations on the effects of projectile impacts to the bones of various animals (Grundfest, 1945; Horsley, 1894) as well as impacts to soft tissues of animals (Harvey and McMillen, 1947; Harvey et al., 1947, 1962).

In our series we found that impacts to human femurs by 0.406 in. stainless steel spheres produced a greater degree of damage than impacts by $0.250 \mathrm{in}$. stainless steel spheres. We concluded that this difference was due to the greater mass and greater diameter of the former. However, on the basis of available information we were unable to determine which factor was more important in imparting energy and thereby causing damage to the target. We know that the larger spheres expend more energy than smaller ones at a given impact velocity. However, because the mass $(4.48 \mathrm{~g})$ of the 0.406 in. sphere is more than four times greater than that of the $0.250 \mathrm{in}$. sphere $(1.04 \mathrm{~g})$, it has a kinetic energy more than four times that of the smaller at a given velocity; but its larger diameter also means it strikes the target with a larger surface $\left(0.259 \mathrm{in}^{2}\right)$, and this factor also may account for the increased energy expenditure of the $0.406 \mathrm{in}$. sphere. In order to separate the effects of projectile mass and projectile diameter on energy transfer to the target, then, it was necessary to keep one factor constant while changing the other and observe the effect on energy loss.

\section{METHOD}

As reported previously (Huelke et al., 1968a), we had measured 220 impacts by $0 \cdot 250$ in. stainless steel spheres on the distal end of embalmed human femurs, using the popliteal plateau as the point of impact so the projectile penetrated the bone in the posteroanterior direction, and 173 impacts by 0.406 in. stainless steel spheres to the same area of other femurs. We then obtained 0.250 in.

\footnotetext{
*Received 27 April 1970.

†This investigation was supported, in part, by PHS Grant UI-00008 from the National Center for Urban and Industrial Health, Cincinnati, Ohio.
} 
spheres composed of Heavimet ${ }^{*}$, a non-deformable tungsten carbide alloy with a specific gravity of $16 \cdot 9$, which increased the mass of the projectile $2 \cdot 18$ times without changing the diameter (the specific gravity of the stainless steel in the previous spheres was 7.75). Using the 0.250 in. Heavimet spheres, we impacted 167 embalmed human femurs in the distal end and compared the results with the previous series.

The method of measurement has been described in a previous report (Huelke et al., 1967). Steel spheres were chosen as projectiles because they eliminate the complications of pitch and yaw of the projectile at impact and they do not lose energy to deformation of the projectile itself at impact. The spheres are fired by a high pressure helium-operated gun built in our laboratory. This muzzle-loading gun can achieve muzzle velocities as low as $100 \mathrm{ft} / \mathrm{sec}$ and as high as $2200 \mathrm{ft} / \mathrm{sec}$ with a maximum deviation in the velocity of 1 per cent. The velocity is regulated by the pressure of helium in the propellant reservoir and by the depth of loading of the projectile into the barrel. The impact velocity (muzzle velocity) is measured by a counter chronograph (Electronic Counters, Inc., Model 464T) switched on by the passage of the projectile through a paper grid and switched off by its passage through a second grid exactly 12 in. beyondthe projectile's velocity is easily calculated from the duration of motion between the two grids. The effect of the paper grids on the projectile's velocity is demonstrably insignificant.

The target femur is firmly mounted $6 \mathrm{ft}$ from the muzzle of the gun, and the impact event is filmed at 14,000 frames/sec by a Wollensak Fastax Model WF-1 high speed camera, using two electronic flash units $\dagger$ synchronized to discharge at impact. These flash units also provide light for photographs of the projectile after exit taken by a Beckman and Whitley Model 326 Dynafax high speed camera running at variable framing rates up to 26,000 frames/sec. Enlarged prints of these Kodak Tri-X Dynafax negatives show the spheres passing two reference points exactly 6 in. apart and permit calculation of the projectile's exit velocity and thus its kinetic energy after impact. The resulting data were subjected to statistical analysis using an IBM 7090 computer - a polynomial regression analysis by the computer generates the values needed to determine the nature of the resulting curves of impact velocity vs. energy loss, whether linear or quadratic, by a one-sided $f$-test and to prove or disprove the statistical difference between any pair of curves.

Before use, each femur was X-rayed and classified by a radiologist $\ddagger$ as 'normal', mildly osteoporotic, or osteoporotic; the energy loss by a given projectile was affected by the degree of calcification of the target femur. The posteroanterior thickness of each femur at the impact site was measured prior to use but was found to have no significant effect on the results of the trials.

Because the proportion of normal, mildly osteoporotic, and osteoporotic femurs in a sample does affect the shape of the final curve best fitting the entire sample, we randomly deleted 28 tests from the total of 220 femurs impacted by $0.250 \mathrm{in}$. steel spheres reported previously (Huelke et al., 1968a). This deletion changed the ratio of normal to mildly osteoporotic to osteoporotic bones from 84: $75: 61$ to $84: 75: 33$; the latter almost exactly agrees with the ratio of $73: 65: 29$ obtained with 0.250 in. Heavimet spheres. Thus, the samples of femurs used in each series were as similar as possible within the limits of determination.

\section{RESULTS}

In a prior publication (Huelke et al., 1968a) we compared the effects of impacts by 0.250 in. steel spheres on the distal end of 50 fresh,

\footnotetext{
* General Electric Company.

†Beckman and Whitley Model 358.

$\ddagger$ Our thanks to A. F. Lalli, M. D. . Department of Radiology, for his invaluahle diagnostic assistance.
} 
unembalmed human femurs and of $220 \mathrm{em}$ balmed human femurs. The curves best fitting the data derived from these series were not significantly different; indeed, they appeared almost identical, suggesting strongly that the embalmed human bones do provide a valid experimental model for the response of human bones to projectile impacts in vivo.

Figure 1 displays the results of 192 impacts on the distal end of embalmed human femurs by $0.250 \mathrm{in}$. steel spheres, 167 impacts by 0.250 in. Heavimet spheres, and 173 impacts by 0.406 in. steel spheres (see also Table 1 ). The curves fitting all three individual sets of data are parabolas to a level of confidence of $p<0.01$. The difference between each curve for $0.250 \mathrm{in}$. spheres and for the $\mathbf{0 . 4 0 6}$ in. steel sphere impacts is statistically significant at the $p<0.01$ level, but there is no statistically significant difference between the curves for 0.250 in. steel spheres and 0.250 in. Heavimet spheres.* Figure 1 clearly shows, however, almost no visible difference in the two curves at velocities of $1250 \mathrm{ft} / \mathrm{sec}$ and over, although there is a slight difference between them at velocities below $1250 \mathrm{ft} / \mathrm{sec}$. The possible significance of this difference at low velocities will be discussed later.

Figure 2 shows the curves best fitting the results of impacts by the three types of spheres on normal, mildly osteoporotic, and osteoporotic femurs. The curve best fitting results from impacts on normal femurs by 0.250 in. Heavimet spheres is a parabola with a statistical level of confidence of $p<0.05$, while the curves for mildly osteoporotic and osteoporotic femurs are parabolas to a level of confidence of $p<0.001$. The curves best fitting data for impacts by $0.250 \mathrm{in}$. steel spheres on normal bones and by $0.406 \mathrm{in}$. steel spheres on normal and on osteporotic femurs were all parabolas to the $p<0.01$ level of confidence. Curves best fitting data for impacts by $\mathbf{0 . 2 5 0}$ in. steel spheres on mildly osteoporotic and on osteo- porotic femurs and by 0.406 in. steel spheres on mildly osteoporotic bones were all parabolas to the $p<0.001$ level of confidence.

Again, no significant difference is apparent between impacts by 0.250 in. steel and Heavimet spheres, although both differ markedly from results with 0.406 in. spheres.

The damage produced by impacts of 0.250 in. Heavimet spheres on embalmed human femurs was similar in all respects to that produced by impacts of 0.250 in. steel spheres with the same impact velocity. This damage has been described previously (Huelke et al., 1968a).

\section{DISCUSSION}

The data suggest that an increase by a factor of 2.18 in the mass (and, therefore, $2.18 \mathrm{X}$ in the kinetic energy) of a projectile does not at all increase the amount of energy it expends at a given impact velocity, nor does it (visibly, at least) increase the amount of damage produced. The Heavimet spheres in this study lost almost exactly the same amount of energy as the 0.250 in. steel spheres at the same impact velocity, and produced the same degree of damage to the distal end of the femur.

However, when the distal end of the femur is impacted at the same velocity by a 0.406 in. steel sphere, which is both 4.3 times as heavy and strikes with 2.64 times greater surface area as the 0.250 in. steel sphere, the energy loss is clearly much higher with the larger sphere and the damage produced is also much greater. The present study, therefore, provides strong evidence that by far the most important factor in determining energy expenditure and thus damage production by the projectile at any given impact velocity is projectile diameter, not projectile mass. The mechanism which explains this finding has already been discussed (Huelke et al., 1967): it is the cavitation phenomenon. As the projectile passes

\footnotetext{
*The significant difference between the curves indicates that the increased mass and diameter of the larger sphere produced a statistically significant increase in encrgy imparted to the target by the projectile.
} 


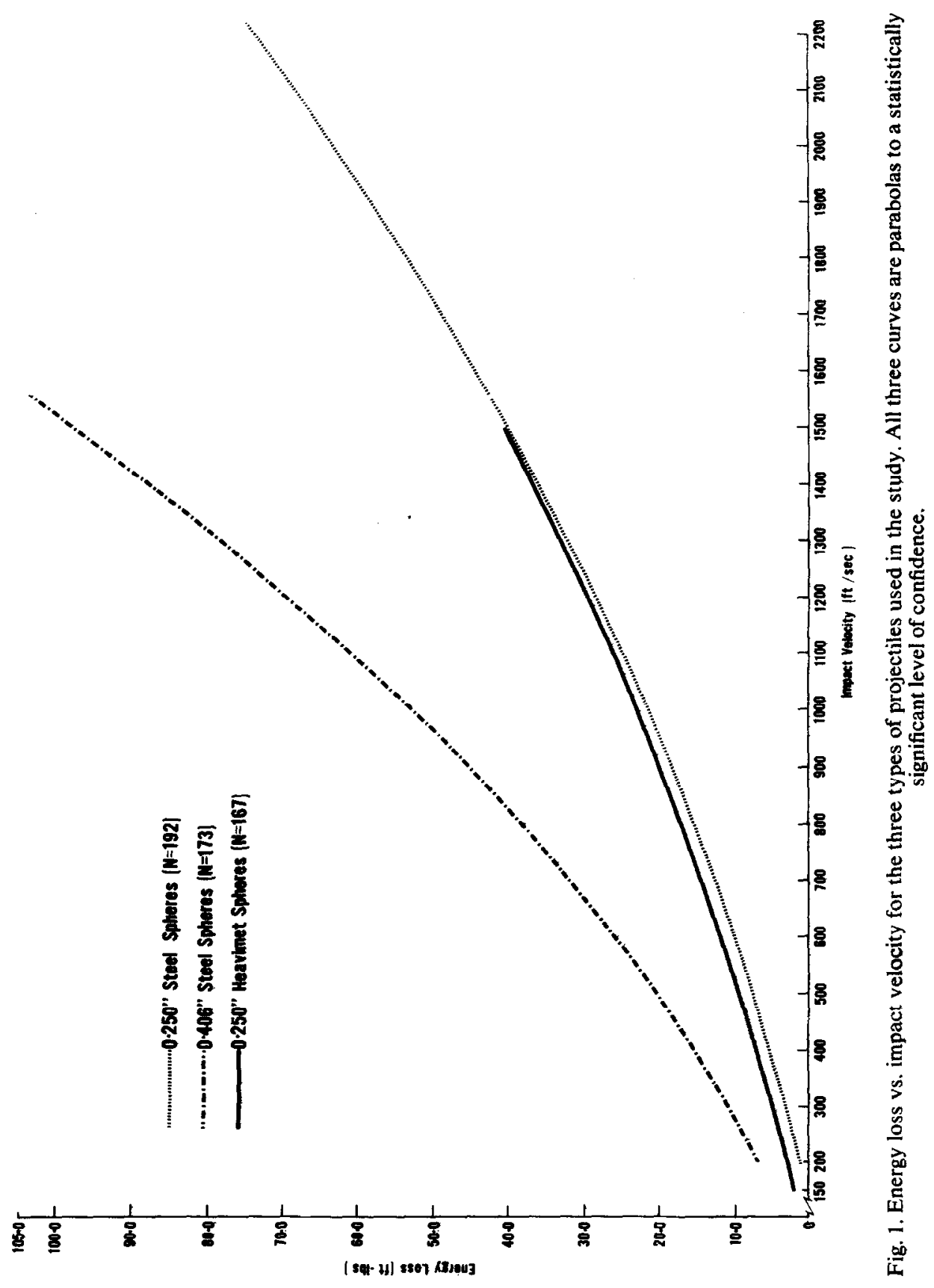




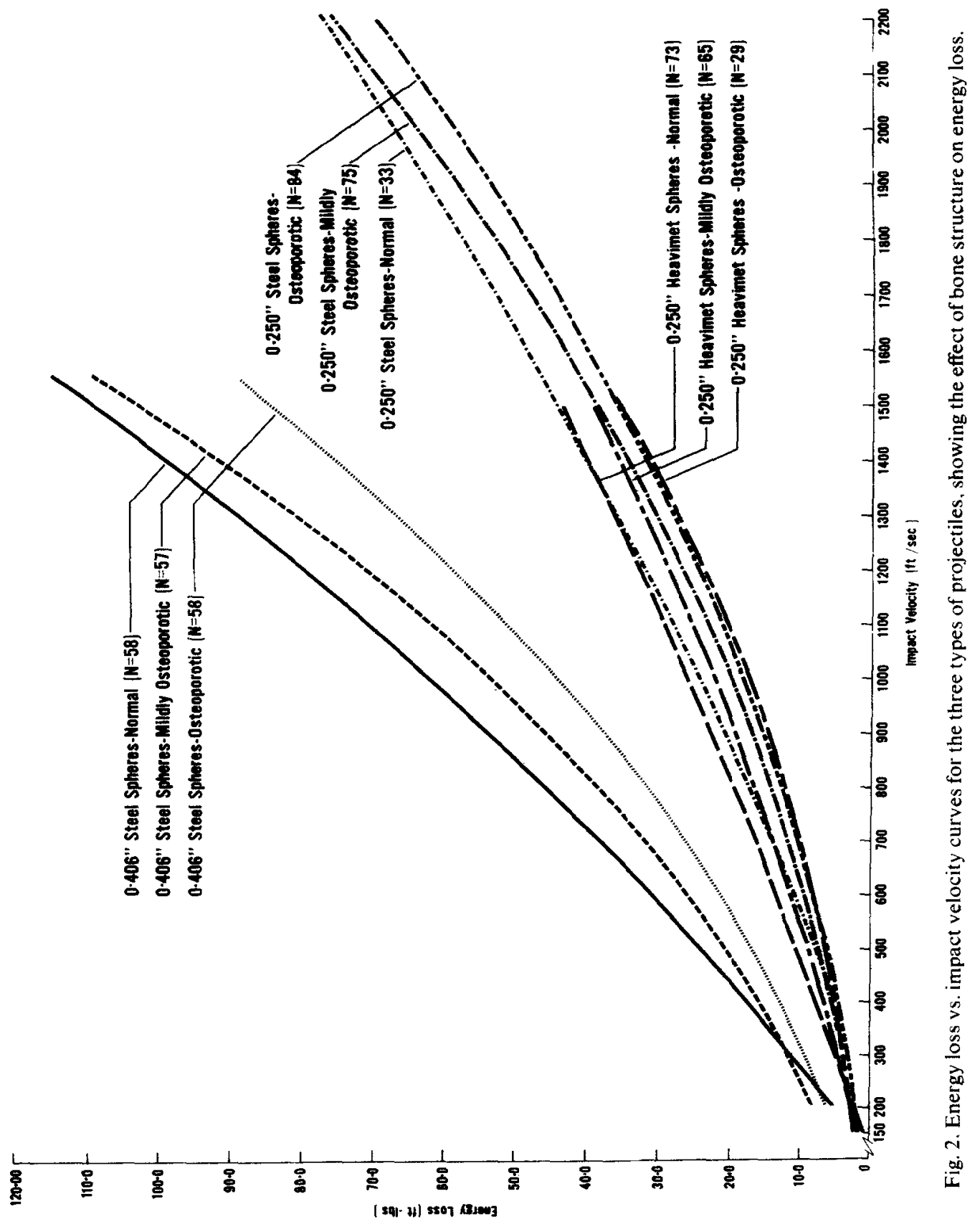


through the target it pushes the substance of the target aside at high velocity, forming 'shock waves' or 'secondary missiles' which magnify the damage far beyond the simple drilling effect of the projectile itself. The larger the surface area presented to the target by the on-rushing projectile, the greater the magnitude of the 'shock wave' produced. Thus the eventual total energy expenditure by the projectile is greater (through increased contact and thus more friction between projectile and target) and, therefore, damage produced is increased significantly. Increased mass of the projectile seems to play little role in increasing energy expenditure at those velocities where the cavitation effect is operative because increased mass does not appreciably increase the magnitude of the cavitation.

Previous work (Huelke et al., 1967 and 1968a) has shown, however, that the effect of cavitation doesn't clearly appear until the impact velocity is $600 \mathrm{ft} / \mathrm{sec}$ with 0.406 in. spheres and $800 \mathrm{ft} / \mathrm{sec}$ with $0.250 \mathrm{in}$. spheres. Thus it may be that the visible difference between the curves in Fig. 1 for $\mathbf{0 . 2 5 0}$ in. steel and Heavimet spheres at velocities below $1250 \mathrm{ft} / \mathrm{sec}$ is due to the lack of significant cavitation effect at low velocity. The sphere with the greater mass and thus greater kinetic energy might, therefore, expend more energy at low velocity; but since there is no statistical difference between the two curves at velocity loss then $1250 \mathrm{ft} / \mathrm{sec}$, this speculation cannot be substantiated at present.

In summary, then, these data strongly indicate that at velocities where the previously described cavitation effect is operative, projectile diameter is a far more important factor influencing energy expenditure at a given velocity than is projectile mass. Therefore, the larger projectile will do the most damage to a target at a given impact velocity, although an

Table 1

\begin{tabular}{rccc}
\hline & \multicolumn{3}{c}{ Energy loss (ft-lb) } \\
\cline { 3 - 4 } $\begin{array}{c}\text { Impact } \\
\text { velocity } \\
\text { (ft/sec) }\end{array}$ & $\begin{array}{c}0 \cdot 250 \text { in. } \\
\text { steel spheres }\end{array}$ & $\begin{array}{c}0 \cdot 250 \text { in. } \\
\text { heavimet spheres }\end{array}$ & $\begin{array}{c}0 \cdot 406 \text { in. } \\
\text { steel spheres }\end{array}$ \\
\hline & & 1.8 & \\
150 & & $2 \cdot 7$ & $7 \cdot 0$ \\
200 & $1 \cdot 3$ & $3 \cdot 7$ & $9 \cdot 0$ \\
250 & $2 \cdot 3$ & $4 \cdot 8$ & $12 \cdot 2$ \\
300 & $3 \cdot 3$ & $7 \cdot 0$ & $15 \cdot 5$ \\
400 & $5 \cdot 4$ & $9 \cdot 3$ & $19 \cdot 9$ \\
500 & $7 \cdot 6$ & $11 \cdot 8$ & $25 \cdot 9$ \\
600 & $10 \cdot 0$ & $14 \cdot 4$ & $32 \cdot 0$ \\
700 & $12 \cdot 7$ & $17 \cdot 2$ & $38 \cdot 3$ \\
800 & $15 \cdot 7$ & $20 \cdot 0$ & $45 \cdot 4$ \\
900 & $18 \cdot 5$ & $23 \cdot 1$ & $52 \cdot 9$ \\
1000 & $21 \cdot 7$ & $26 \cdot 2$ & $61 \cdot 0$ \\
1100 & $25 \cdot 1$ & $29 \cdot 3$ & $69 \cdot 5$ \\
1200 & $28 \cdot 6$ & $33 \cdot 0$ & $78 \cdot 1$ \\
1300 & $32 \cdot 3$ & $36 \cdot 6$ & $87 \cdot 8$ \\
1400 & $36 \cdot 3$ & $40 \cdot 3$ & $98 \cdot 1$ \\
1500 & $40 \cdot 1$ & & $108 \cdot 6$ \\
1600 & $44 \cdot 7$ & & \\
1700 & $49 \cdot 1$ & & \\
1800 & $53 \cdot 6$ & & \\
1900 & $58 \cdot 5$ & & \\
2000 & $63 \cdot 6$ & & \\
2100 & $69 \cdot 0$ & & \\
2200 & $74 \cdot 3$ & & \\
\hline
\end{tabular}


increase in the impact velocity of the projectile will also produce a very substantial increase in energy expenditure and damage produced.

\section{REFERENCES}

Grundfest, H. (1945) Penetration of steel spheres into bone. Missiles Casualty Rep. No. 10. National Research Council, Division of Medical Science, Office of Research and Development.

Harvey, E. N., Korr, J. M., Oster, G. and McMillen, J. H. (1947) Secondary damage in wounding due to pressure changes accompanying the passage of high velocity missiles. Surgery 21, 218-239.

Harvey, E. N. and McMillen, J. H. (1947) An experimental study of shock waves resulting from the impact of high velocity missiles on animal tissues. $J$. exp. Med. 85, 321-328.

Harvey, E. N., McMillen, J. H., Butler, E. G. and Puckett, W. O. (1962) In Wound Ballistics. (Edited by J. B. Coates and J. C. Beyer), Chap. 3, pp. 143-236. Office of the Surgeon General, Department of the Army, Washington, D.C.

Horsley, V. (1894) The destructive effects of projectiles. Not. Proc. R. Inst. Great Britain 14, 228-238.

Huelke, D. F., Buege, L. J. and Harger, J. H. (1967) Bone fractures produced by high velocity impacts. $A m$. J. Anat. 120, 123-131.

Huelke. D. F., Harger, J. H., Buege, L. J., Dingman, H. G. and Harger, D. R. (1968a). An experimental study in bio-ballistics: femoral fractures produced by projectiles - I. Distal impacts. J. Biomechanics 1, 97-105.

Huelke, D. F.. Harger, J. H.. Buege, L. J. and Dingman, H. G. (1968b) An experimental study in bio-ballistics: femoral fractures produced by projectiles-II. Shaft impacts. J. Biomechanics 1, 313-321. 